Supplementary Information

\title{
Imaging-based Optofluidic Biolaser Array Encapsulated with Dynamic Living Organisms
}

Xuerui Gong ${ }^{1+}$, Shilun Feng ${ }^{2 *+}$, Zhen Qiao ${ }^{1}$, and Yu-Cheng Chen ${ }^{1,3 *}$

1 School of Electrical and Electronics Engineering, Nanyang Technological University, 50 Nanyang Ave. 639798, Singapore

2 State Key Laboratory of Transducer Technology, Shanghai Institute of Microsystem and Information Technology, Chinese Academy of Science, Shanghai, 200050, China

3 School of Chemical and Biomedical Engineering, Nanyang Technological University, 62 Nanyang Dr. 639798, Singapore

+ Equal Contribution

*Correspondence Email: shilun.feng@mail.sim.ac.cn

*Correspondence Email: yucchen@,ntu.edu.sg 


\section{Table of contents}

Figure S1. Reflective profile of mirror and optical setup. (Page S-3)

Figure S2. Viability confirmation of E.coli after printing. (Page S-4)

Figure S3. COMSOL simulation of droplet resonator. (Page S-5)

Figure S4. Capability and reliability of printer. (Page S-6)

Figure S5. Optical properties of E.coli. (Page S-7)

Figure S6. Statistic of E.coli quantity in 30 droplets. (Page S-8)

Figure S7. Fluorescent images taken at different Z-planes of droplet with 4 E.coli. (Page S-9)

Figure S8. $\quad$ Fluorescent images of E.coli before and after adding Ampicillin. (Page S-10)

Figure S9. Integrated pixel intensity change of laser image over time. (Page S-11)

Figure S10. Comparison between volatility of water/glycerol and water droplets. (Page S-12)

Figure S11. Comparison of fluorescence intensity in supernatant and substrate. (Page S-13) 


\section{SI Figures}

a

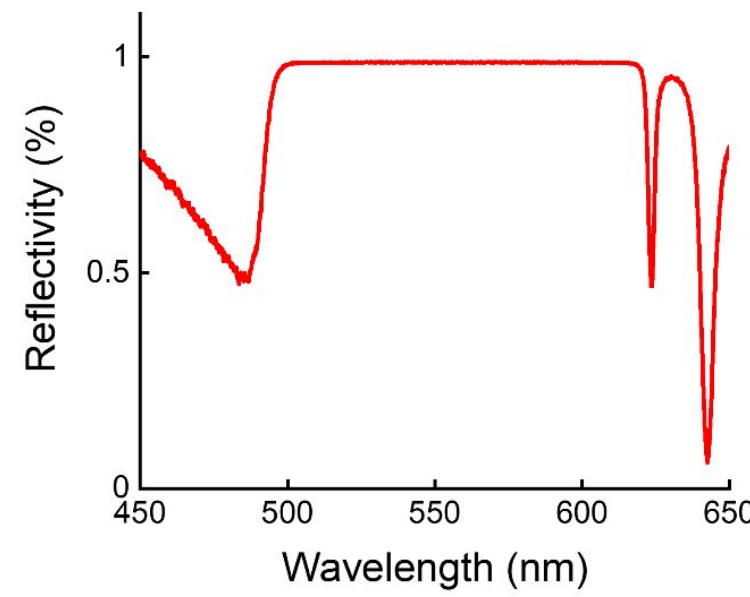

b

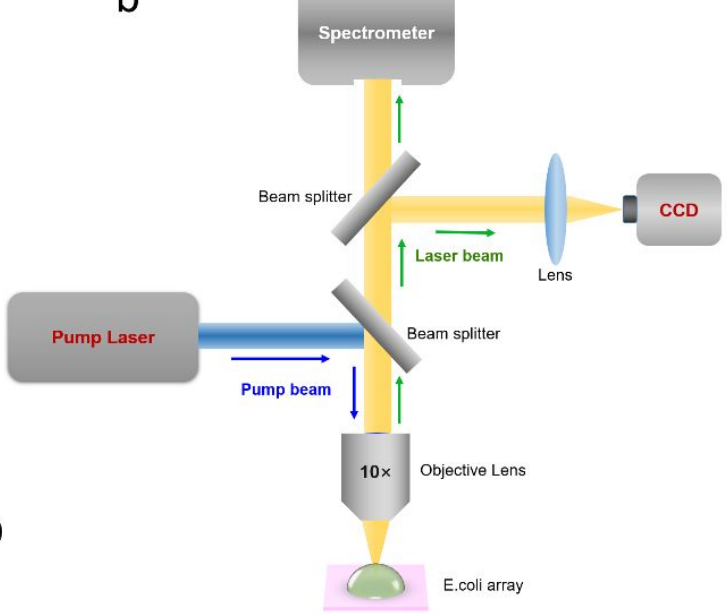

Figure. S1 (a) Reflective profile of the dielectric mirror used in experiment. (b) Schematic diagram of the optical system. 
a

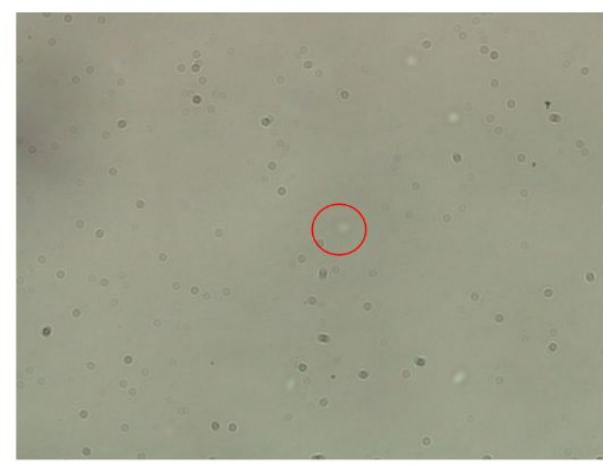

Before printing b

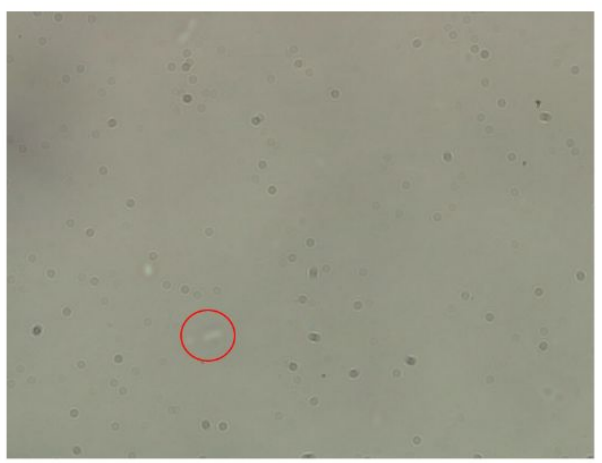

After printing

Figure. S2 White light image of E.coli mixing with Trypan blue. (a) Before printing and (b) after printing. 
a

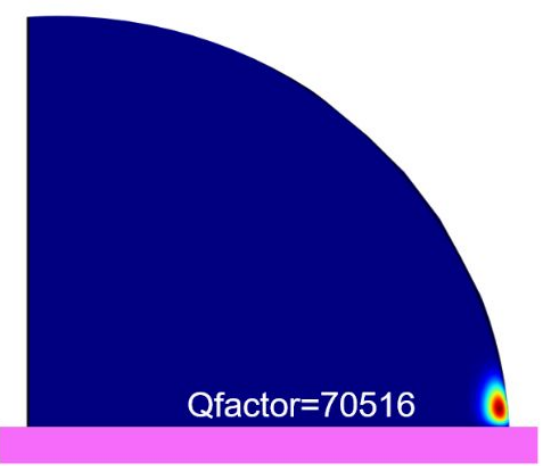

High reflective mirror

C

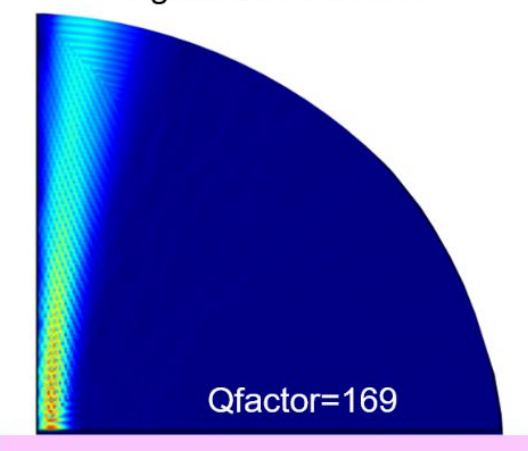

Low reflective mirror b

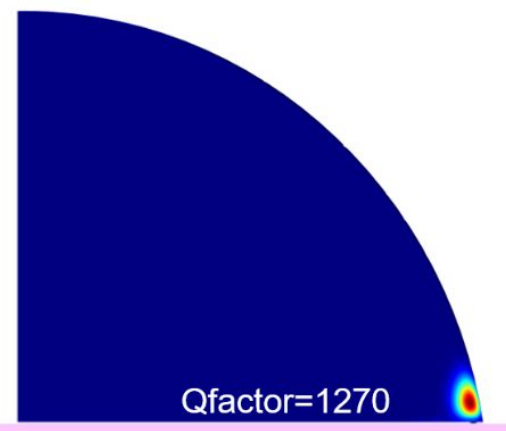

Low reflective mirror

Figure. S3 Simulated 3D electrical field distribution of WGM and FP resonant cavity mode for a $40 \mu \mathrm{m}$ droplet. (a) WGM mode on a high reflective bottom mirror. (b) WGM mode on a low reflective mirror. (c) FP cavity mode on a high reflective mirror. 

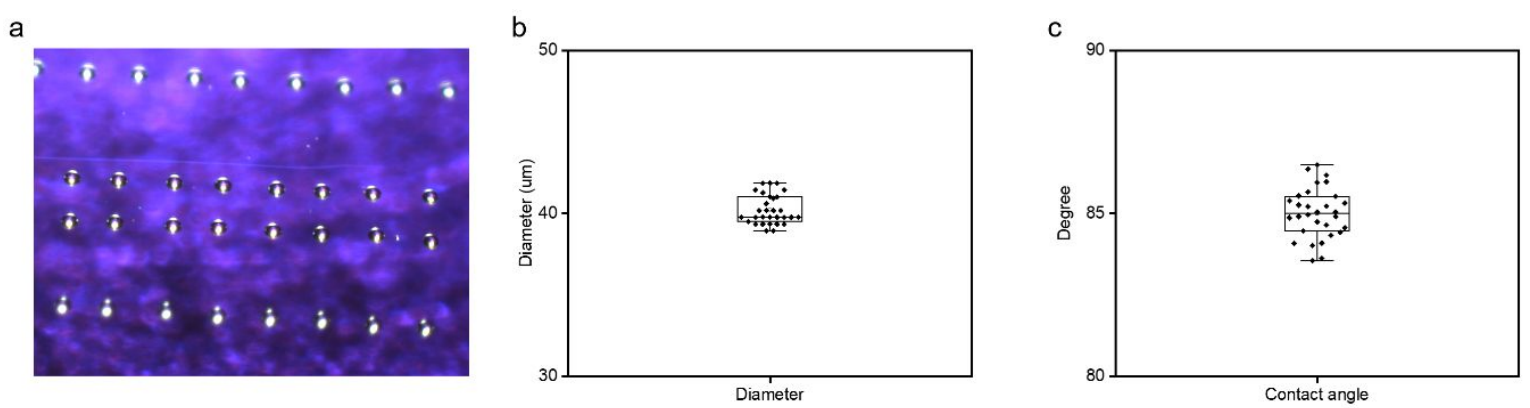

Figure. S4 (a) Side view image of the printed array. (b-c) Statistics on the droplets sizes and contact angle. 

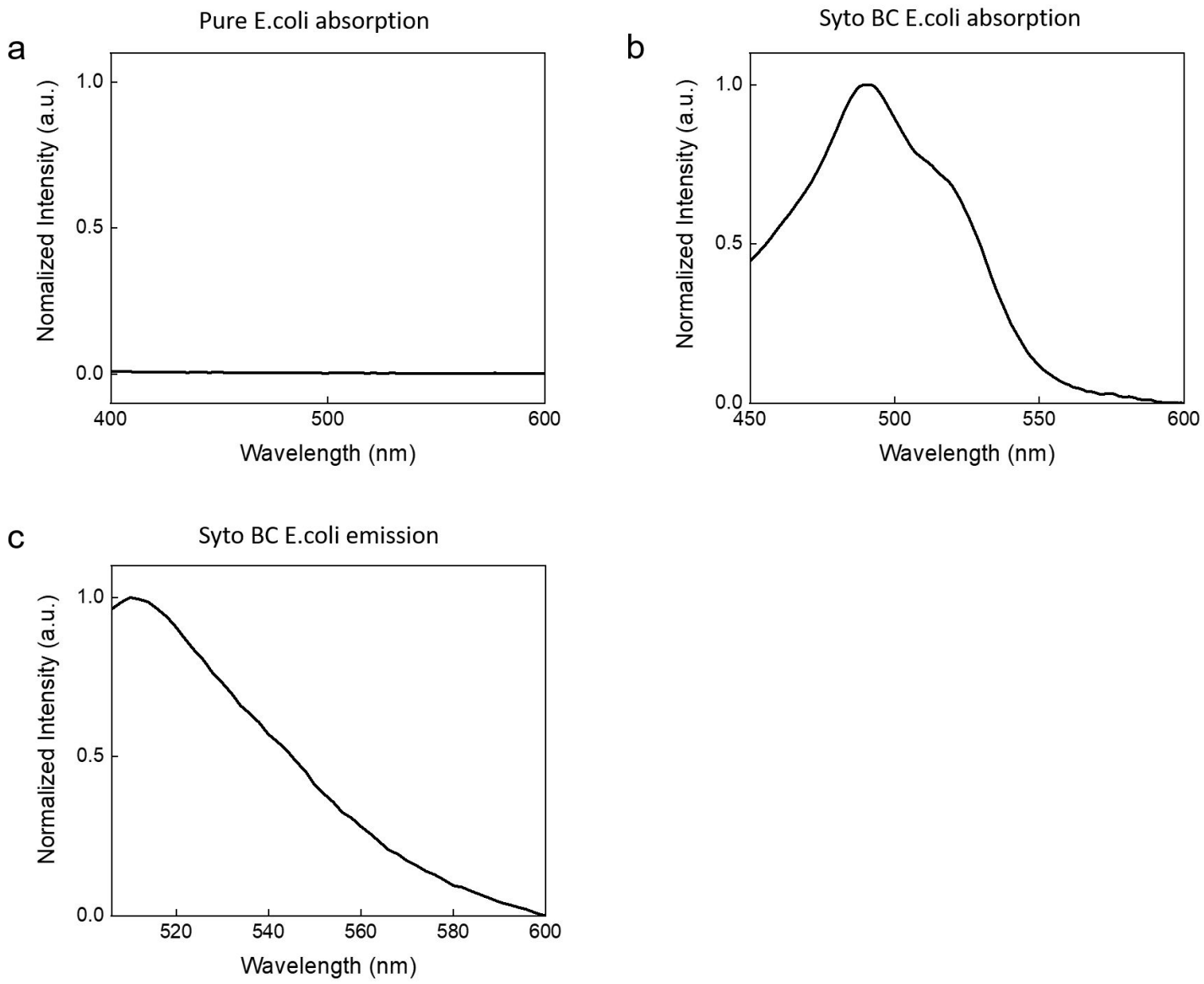

Figure. S5 (a) Absorption spectrum of unstained E.coli. (b) Absorption spectrum of Syto-BC labelled E.coli. (c) Emission spectrum of Syto-BC labelled E.coli. 


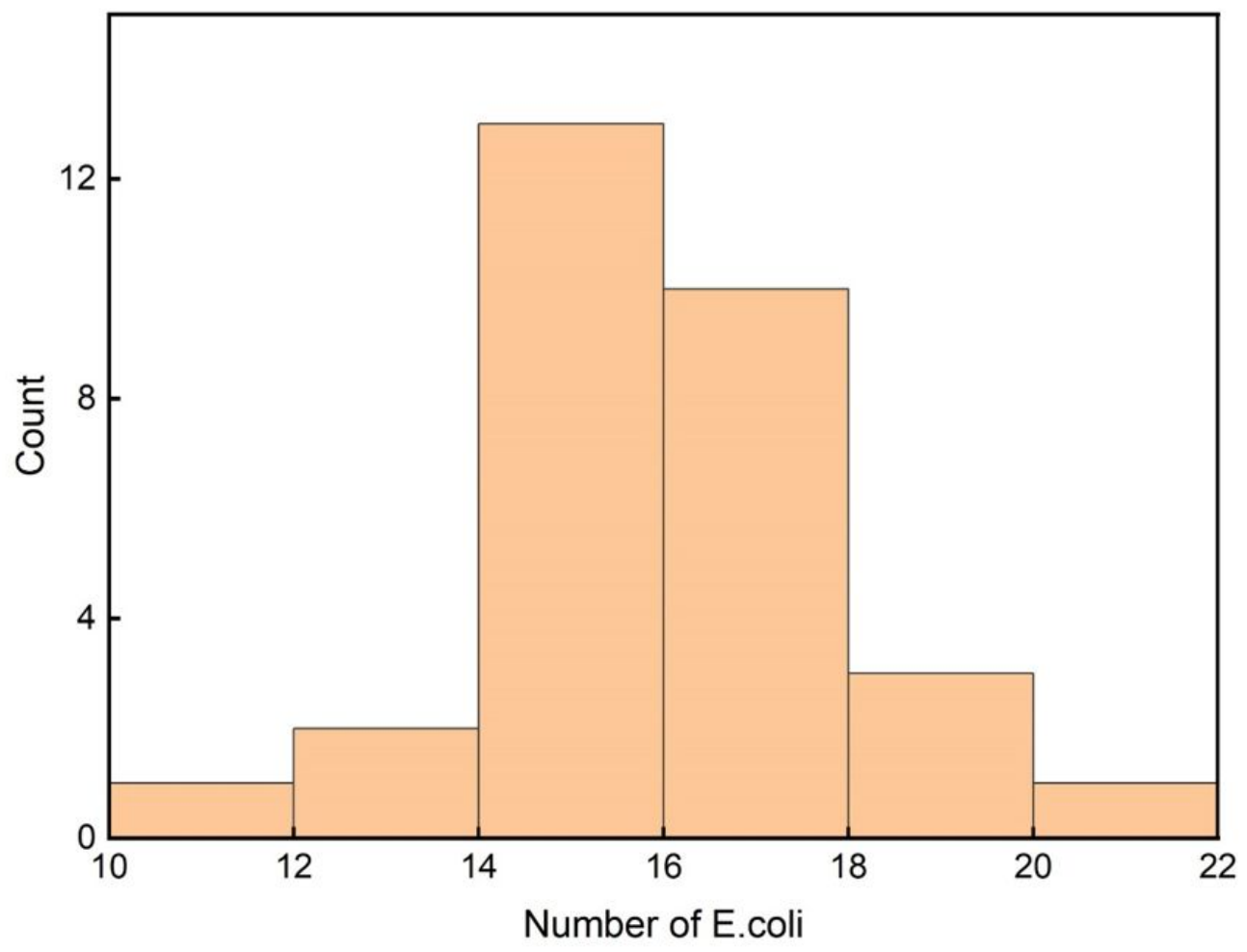

Figure. S6 Statistic of E.coli counts inside droplets. Here we showed the results after calculating 30 individual droplets. The distribution was highly concentrated around 16 E.coli which is consistent with the setting in printer. 




$-2245$



$-2241$

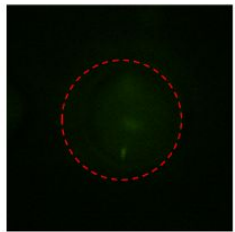

$-2237$

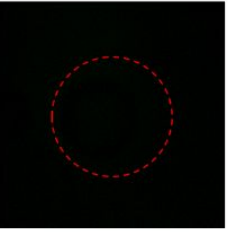

$-2233$

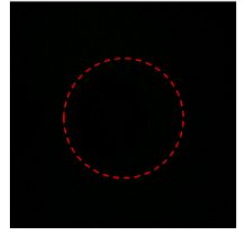

$-2229$



$-2225$

Figure. S7 Fluorescence images recorded from droplet with 4 E.coli on different Zfocus planes. All droplet size, $40 \mu \mathrm{m}$. Numbers below presents corresponding $\mathrm{Z}$ axis (depth) coordinates each image on Z-axis (unit: microns). 
a

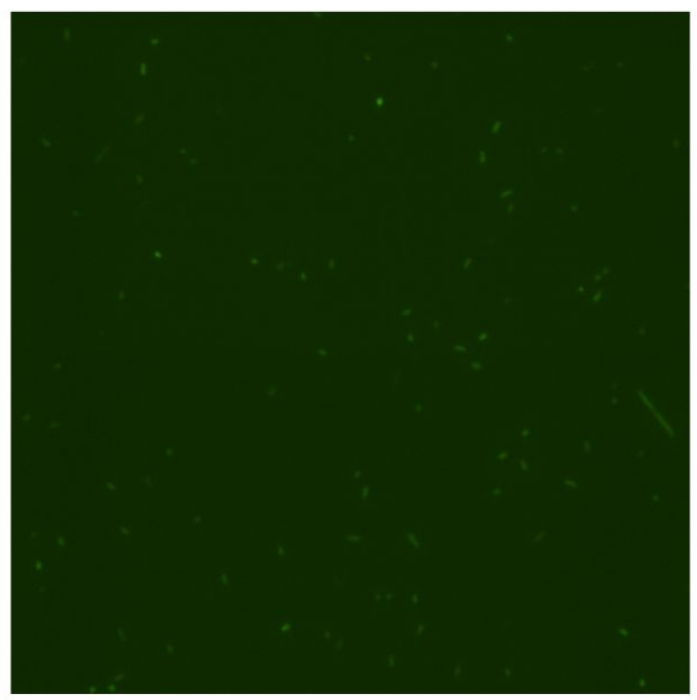

b

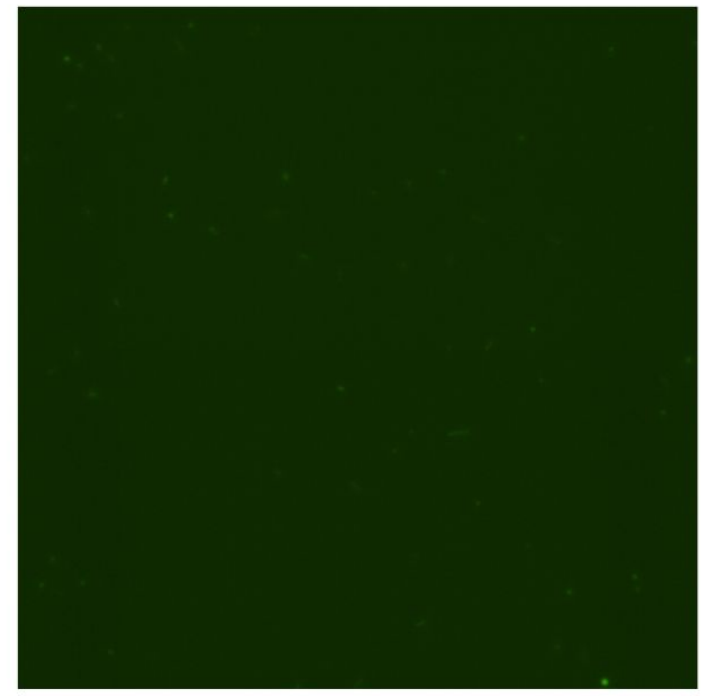

Figure. S8 (a) Fluorescent image of E.coli before adding Ampicillin. (b) Fluorescent image of E.coli after adding Ampicillin for 15 mins. 


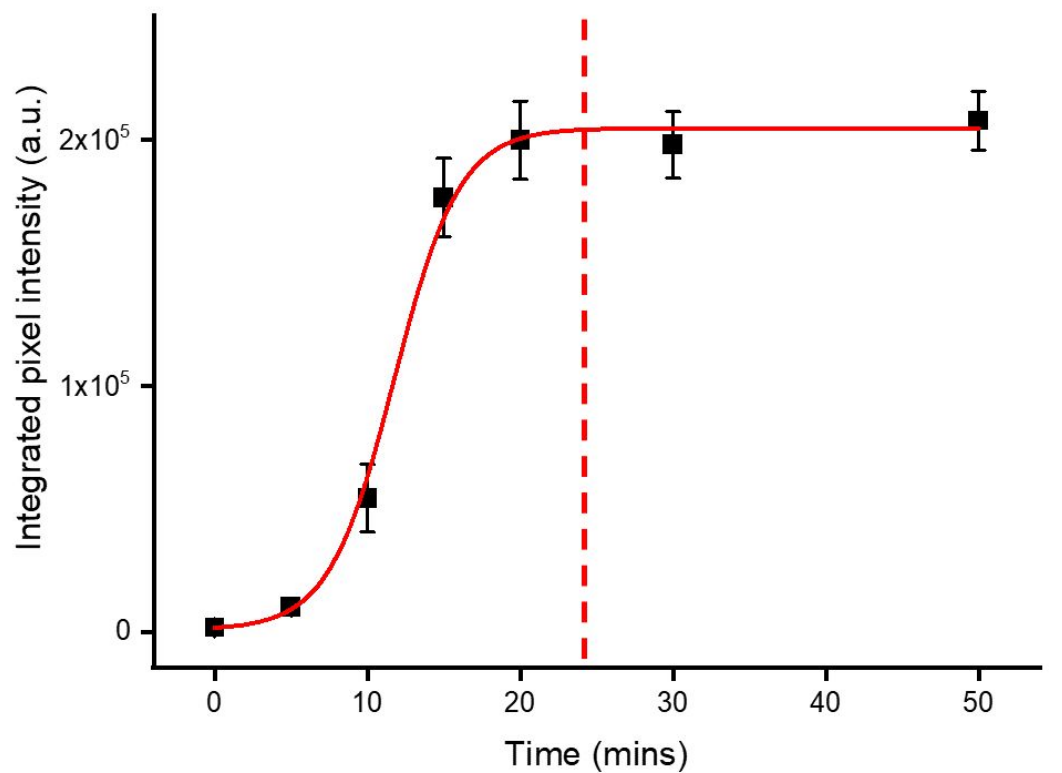

Figure. S9 Integrated pixel intensity of lasing images after adding E.coli for 0, 5, 10, $15,20,30,50$ mins. The intensity saturated around 23 mins. 


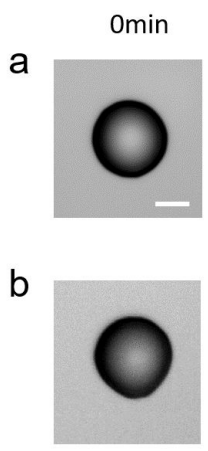

Os

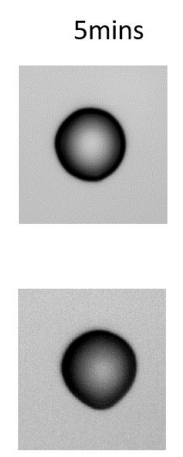

1s
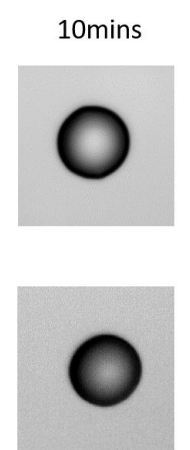

$2 s$
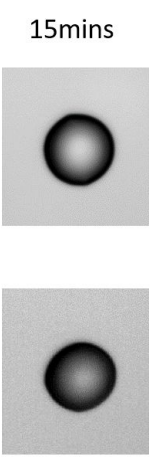

$3 s$ 20mins


Pure water

$4 s$

Figure. S10 Top view profiles of (a) water/glycerol droplet and (b) pure water droplet over time. Scale bar, $20 \mu \mathrm{m}$. 
a

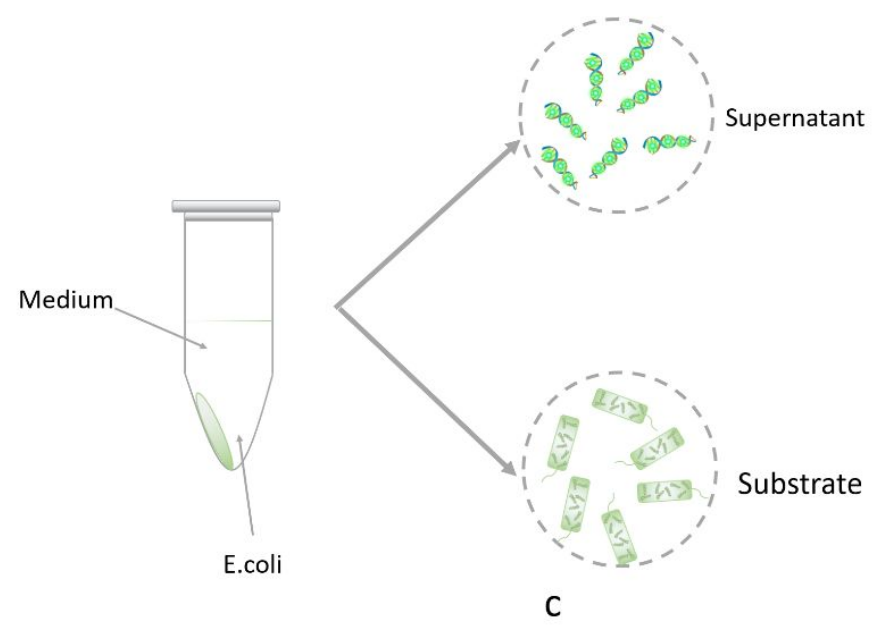

b

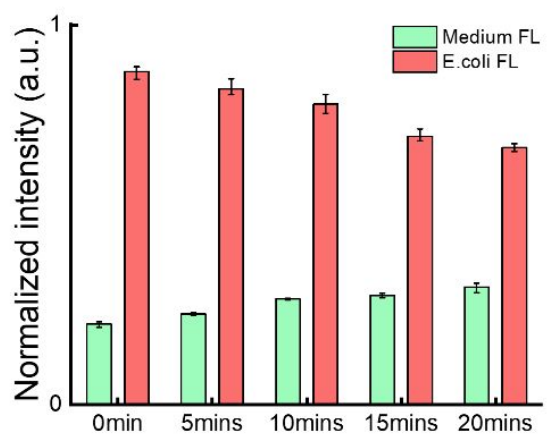

C

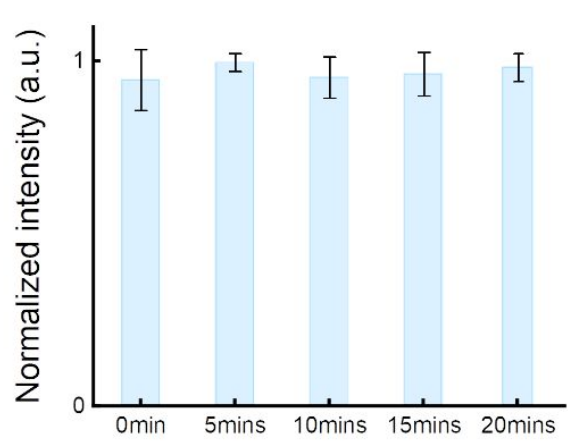

Figure. S11 (a) Schematic diagram showing the substances in supernatant (fluorescent DNA) and substrate (E.coli) after centrifuge. (b) Fluorescence intensity measured from supernatant and substrate (after centrifuge) under different treatment periods. (c) Control group: Fluorescence intensity from original bio-ink after adding Ampicillin under different treatment periods (without centrifuge). All the intensities were based on the readout from fluorescence spectrometer. 